\title{
The Wess-Zumino Term and Fermionic Solitons
}

\author{
T. R. Ramadas
}

School of Mathematics, Tata Institute of Fundamental Research, Bombay 400 005, India

\begin{abstract}
In [1,2] Witten derived important consequences of the topological structure of $\sigma$-models. We rederive them from the canonical viewpoint and outline a systematic approach to similar questions.
\end{abstract}

\section{Introduction}

In $[1,2]$, Witten derived several striking consequences of the topological structure of $\sigma$-models. In particular he showed that for the $S U(3) \sigma$-model:

i) the Wess-Zumino term in the equation of motion has to occur in integral multiples of a certain fixed expression, and

ii) the solitons are fermionic.

Our object is to rederive these as consequences of a Hamiltonian approach. This clarifies the topological aspect of the problem, and gives a systematic way of studying such questions. The requirement that a certain two-form be integral and thus represent a curvature on a line bundle gives i). The assertion ii) follows from the fact that a certain $S O(3)$ action does not lift to the line bundle.

We should note that our work is similar in spirit to that of Finkelstein and Rubinstein [3] and the more recent papers [4-6]. Other recent references are $[7,8]$. Jain and Wadia are investigating the Hamiltonian structure independently.

In the rest of this section we outline our argument.

Let us start, as Witten does, by considering a charged spinless particle in the field of a magnetic monopole. But instead of trying to write down a Lagrangian we note (see for e.g. [9]) that the equations of motion can be written in a Hamiltonian form by changing the symplectic structure in a way that only involves the magnetic field $F^{1}$,

$$
\left\{p_{i}, p_{j}\right\}=\text { const } \times F_{i j} .
$$

1 This corresponds to the following: The configuration space $M$ is $\mathbb{R}^{3} \backslash\{0\}$. The cotangent bundle $\pi: T^{*} M \rightarrow M$ is the phasespace, and carries a canonical nondegenerate 2-form $\Omega_{0}$, which defines the usual Poisson brackets. Let $\Omega=\Omega_{0}+\pi^{*} F$, where $F$ is the magnetic field 2-form, and check $\Omega$ is again nondegenerate. Then $\Omega$ defines the new symplectic structure 
Now one can quantise the system directly. Geometric quantisation [10], in this special situation gives the following prescription: find a hermitian line bundle (i.e. a complex vector bundle with one-dimensional fibres and an inner product along the fibres) $L$ and a unitary connection $A$ on $L$ so that $F$ is its curvature. The Hilbert space of states $\mathscr{H}$ is then the space of square integrable sections of $L$ and vector fields on $\mathbb{R}^{3} \backslash\{0\}$ act on $\mathscr{H}$ by covariant differentiation so that (1) is implemented as an operator equation.

This gives immediately i) the quantisation of the magnetic charge and ii) the fermionic nature of the system for odd values of the magnetic charge. The curvature integrated over a 2 -sphere has to give the Chern class, an integer - this implies i). As for ii), note that $L$ restricted to a two-sphere $\left(\sim \mathbb{P}_{\mathbb{C}}^{1}\right)$ can be identified with a power $L_{0}$ of the so-called tautological line bundle $L_{0}$ on $\mathbb{P}_{\mathbb{C}}^{1}$ (given by associating to each point the one-dimensional subspace of $\mathbb{C}^{2}$ it represents). Then it is well-known that the action of $S O(3)$ on $\mathbb{P}_{\mathbb{C}}^{1}$ [induced by the action of $S U(2)$ on $\left.\mathbb{C}^{2}\right]$ lifts to an action on $L_{0}$ iff $r$ is even. Thus when $r=1$ (unit magnetic charge, up to sign) only $S U(2)$ acts on $L_{0}$, and hence the state-space carries a half-integral spin.

Let us now turn to the $\sigma$-model described by Witten. It describes an $S U(3)$ valued field $U$, and the equation of motion is

$$
\partial^{\mu} A_{\mu}+\chi \varepsilon^{\mu v \varrho \sigma} A_{\mu} A_{v} A_{\varrho} A_{\sigma}=0,
$$

where $A_{\mu}=U^{+} \partial_{\mu} U$ and $\chi$ is a constant. The second term is the Wess-Zumino term.

The "configuration space" $\mathfrak{M}$ of the theory is the space of maps $S^{3} \rightarrow S U(3)$. This is because finiteness of energy is supposed to force $U$ to go to a constant at spatial infinity. The space $\mathfrak{M}$ falls into components - the classical soliton sectors labelled by $\pi_{3}(S U(3))=\mathbb{Z}$, which we shall label $\mathfrak{M}^{(n)}$. We have then

a) The components are homeomorphic. We have $H^{2}\left(\mathfrak{M}^{(n)}\right)=\mathbb{Z}$, so that the set of isomorphism classes of (unitary) line bundles on $\mathfrak{M}^{(n)}$ is again labelled by $\mathbb{Z}$. Let $\mathscr{L}^{(i)}$ denote the line-bundle with this integer (the Chern class) equal to $i$.

b) The equation of motion (2) can be derived by changing the symplectic structure on $T^{*} \mathfrak{M}$ by a curvature term $\chi \mathscr{J}$ (analogous to the magnetic field) pulled back from $\mathfrak{M}$.

c) $\mathscr{J}$ represents the "same" cohomology class on each $\mathfrak{M}^{(i)}$. [This makes sense because of (a).]

d) Quantisation would require that $\chi \mathscr{J}$ represent an integral cohomology class, so as to be a curvature of a connection on a line bundle. If we require that it represents a generator of $H^{2}\left(\mathfrak{M}^{(i)}\right)$ this fixes the least value $\chi_{0}$ of $\chi: \chi_{0} \mathscr{J}$ is a curvature on $\mathscr{L}^{(1)}$.

e) The group of spatial rotations $S O(3)$ acts on $\mathfrak{M}^{(i)}$. Its action lefts to $\mathscr{L}^{(j)}$ for $j$ even, and to $\mathscr{L}^{(j)}$ for $j$ odd if $i$ is even. It only lifts to an $S U(2)$ action otherwise. Thus, if $\chi=($ odd integer $) \times \chi_{0}$, the one-soliton sector is fermionic.

The rest of this paper is organised as follows. Section 2 describes the Hamiltonian formulation of the $\sigma$-model, and the modification needed to take the Wess-Zumino term into account.

Section 3 is devoted to some topological results on the $\mathfrak{M}^{(n)}$ and to deriving the "quantisation" of $\chi$. We conclude this section with remarks on the $S U(2) \sigma$-model. In Sect. 4 we have the results on the spin of the quantised theory. 
Because of the infinite dimensional situation, the differential geometric statements below are only formally true, and these are marked with an asterisk. The purely topological statements are true when spaces of maps have the compactopen topology.

\section{The $\sigma$-Model}

We consider space to be compactified to give $S^{3}=S U(2)$. We will denote by $\left\{\partial_{i}\right\}_{i=1,2,3}$ a set of orthonormal, left invariant vector-fields. The volume form on $S^{3}$ will be denoted by $\tau$.

The configuration space of the theory is the space of maps $S^{3} \rightarrow S U(3)$. This is formally a Lie group, and its Lie algebra $\mathscr{G}$ is the space of maps $S^{3} \rightarrow s u(3)$, the Lie algebra of $S U(3)$. We have the tangent bundle

$$
\begin{gathered}
T \mathfrak{M}=\mathfrak{M} \times \mathscr{G}, \\
(U, \delta U) \mapsto\left(U, U^{+} \delta U \equiv A_{t}\right) .
\end{gathered}
$$

We shall define Hamiltonian dynamics on $T \mathfrak{M}$. [It should, of course, be defined on $T^{* \mathfrak{M}}$, but we have $\mathscr{G} \sim \mathscr{G}^{*}$ by means of the inner product $\left(A_{t}, A_{t}^{\prime}\right)=-\int \operatorname{Tr}\left(A_{t} A_{t}^{\prime}\right) \tau$.]

Take as Hamiltonian the function

$$
H\left(U, A_{t}\right)=-\frac{1}{2} \int \operatorname{Tr}\left(A_{t}^{2}+\sum_{i} A_{i}^{2}\right) \tau,
$$

where $A_{i}=U^{+} \partial_{i} U$. (Note that $A \equiv \sum_{i} A_{i} d x^{i}$ is the pull-back of the Maurer-Cartan form by the map $U$ ).

We have

$$
T(T \mathfrak{M})=\underbrace{\mathfrak{M} \times \mathscr{G}}_{T \mathfrak{M}} \underbrace{\times \mathscr{G} \times \mathscr{G}}_{T \mathscr{G}}
$$

with coordinates $\left(U, \alpha, A_{t}, \beta\right)$ and we define the symplectic structure by the 2 -form

$$
\Omega_{\left(U, A_{t}\right)}\left\{(\alpha, \beta),\left(\alpha^{\prime}, \beta^{\prime}\right)\right\}=-\int \operatorname{Tr}\left(\beta \alpha^{\prime}-\beta^{\prime} \alpha-A_{t}[\alpha, \alpha]\right) \tau+\chi \mathscr{J}\left\{(\alpha, \beta),\left(\alpha^{\prime}, \beta^{\prime}\right)\right\},
$$

with

$$
\begin{aligned}
\mathscr{J}\left\{(\alpha, \beta),\left(\alpha^{\prime}, \beta^{\prime}\right)\right\}= & \int \operatorname{Tr}\left(\alpha A_{i} A_{j} A_{k} \alpha^{\prime}-A_{i} \alpha A_{j} A_{k} \alpha^{\prime}\right. \\
& \left.+A_{i} A_{j} \alpha A_{k} \alpha^{\prime}-A_{i} A_{j} A_{k} \alpha \alpha^{\prime}\right) \varepsilon^{i j k} \tau .
\end{aligned}
$$

We will see later that $\mathscr{J}$ is closed. Note that it only involves $\left(\alpha, \alpha^{\prime}\right)$ and is thus pulled back from $\mathfrak{M}$.

We now verify that $\Omega$ gives the equation of motion (2). Note first that

$$
d H\left(\alpha^{\prime}, \beta^{\prime}\right)=-\int \operatorname{Tr}\left(A_{t} \beta^{\prime}+A_{i} \delta A_{i}\left(\alpha^{\prime}\right)\right) \tau,
$$

where $\delta A_{i}\left(\alpha^{\prime}\right)=\partial_{i} \alpha^{\prime}+\left[A_{i}, \alpha^{\prime}\right]$, so that

$$
d H\left(\alpha^{\prime}, \beta^{\prime}\right)=-\int \operatorname{Tr}\left(A_{t} \beta^{\prime}+A_{i} \partial_{i} \alpha\right) \tau .
$$


The Hamiltonian vector field $\left(\alpha_{H}, \beta_{H}\right)$ is defined by

$$
d H\left(\alpha^{\prime}, \beta^{\prime}\right)=-\Omega\left\{\left(\alpha_{H}, \beta_{H}\right),\left(\alpha^{\prime}, \beta^{\prime}\right)\right\} .
$$

Comparing coefficients of $\alpha^{\prime}$ and $\beta^{\prime}$, we get

$$
\begin{gathered}
\alpha_{H}=A_{t}, \\
\beta_{H}=d^{*} A-\chi\left(\alpha_{H} A_{i} A_{j} A_{k}-A_{i} \alpha_{H} A_{j} A_{k}\right. \\
\left.+A_{i} A_{j} \alpha_{H} A_{k}-A_{i} A_{j} A_{k} \alpha_{H}\right) \varepsilon^{i j k}
\end{gathered}
$$

[where $d^{*}$ is the adjoint of $d$. On $\mathbb{R}^{3}$ the term $d^{*} A$ would be equal to $\partial_{i} A_{i}$ $\left.=\partial_{i}\left(U^{+} \partial_{i} U\right)\right]$. It is easily seen that this reproduces (2).

Remark 2.1. There is a description of the curvature term $\mathscr{J}$ which makes it clear why it is closed, and will be useful later. Consider the closed form $\Theta=\operatorname{Tr}(w \wedge w \wedge w \wedge w \wedge w)$ on $S U(3)$, where $w$ is the Maurer-Cartan form. Then the evaluation map

$$
\begin{gathered}
\mathfrak{M} \times S^{3} \stackrel{G}{\longrightarrow} S U(3), \\
(U, x) \mapsto U(x),
\end{gathered}
$$

gives by pull-back a closed 5 -form $G^{*} \Theta$ on the product, and $\mathscr{J}$ is obtained by integrating along the fibres of the projection $\left(p_{1}\right)$ to the first factor. Thus

$$
\mathscr{J}=p_{1 *} G^{*} \Theta \text {. }
$$

\section{The Topology of $\mathfrak{M i}^{(n)}$ and the Class of $\mathscr{J}$}

We shall systematically identify $S^{3} \sim S U(2)$. Let as before

$$
\mathfrak{M}=\operatorname{Maps}(S U(2), S U(3)) \text {. }
$$

Then since $\pi_{3}(S U(3)) \sim \mathbb{Z}$ we have $\mathfrak{M}=\bigcup_{n \in \mathbb{Z}} \mathfrak{M}(n)$, where $\mathfrak{M}^{(n)}$ are the path components. We shall denote by I the standard inclusion of $S U(2)$ in $S U(3)$

$$
S U(2) \ni(M) \mapsto\left(\begin{array}{c|c|c}
M & 0 \\
\hline 0 & 0 & 1
\end{array}\right) \in S U(3),
$$

and take the corresponding element of $\pi_{3}(S U(3))$ as the positive generator. We have

Proposition 3.1. The $\mathfrak{M}^{(n)}$ 's are topologically isomorphic. We have

$$
\pi_{1}\left(\mathfrak{M}^{(n)}\right)=0, \quad \pi_{2}\left(\mathfrak{M}^{(n)}\right)=\mathbb{Z} .
$$

Proof. Let $e$ be the identity of $S U(2)$. Evaluation at $e$ gives a map

$$
\mathfrak{M}^{(n)} \stackrel{E}{\longrightarrow} S U(3) \text {. }
$$

Let $\hat{\mathfrak{M}}^{(n)}$ be the fibre above the identity of $S U(3)$. Define a map $T: \mathfrak{M} \rightarrow \mathfrak{M}$ by $T f(x)$ $=f(x) \mathbf{I}(x)$. It is clear that $T$ takes $\mathfrak{M}$ isomorphically to itself, taking $\mathfrak{M}^{(n)}$ to $\mathfrak{M}^{(n+1)}$ 
(Since multiplication in $\pi_{i}(G)$ for $G$ a group can be defined by multiplication in the group itself, see, e.g. [11]). This proves the first statement. The statement about homotopy groups follow from the (trivial) fibration

$$
\begin{gathered}
\mathfrak{M}^{(0)} \\
\downarrow \downarrow \\
S U(3)
\end{gathered}
$$

noting $\pi_{i}\left(\hat{\mathfrak{P}}^{(0)}\right)=\pi_{i+3}(S U(3))$.

Remark 3.2. By Hurewicz and the universal coefficient theorem (together with the above proof), we have $H^{2}\left(\mathfrak{M}^{(n)}\right) \rightarrow H^{2}\left(\hat{\mathfrak{M}}^{(n)}\right)=\mathbb{Z}$.

*Theorem 1. The curvature $\mathscr{J}$ represents the same cohomology class in each $\mathfrak{M}^{(n)}$. If $\chi_{0} \mathscr{J}$ represents a generator of $H^{2}, \chi_{0}=-\frac{i}{2 \pi} \frac{1}{240 \pi^{2}}$.

Proof. As pointed out in Remark 2.1, $\mathscr{J}$ is obtained by pulling back $\Theta=\operatorname{Tr}(w \wedge \ldots w)$ under the evaluation map

$$
\mathfrak{M} \times S U(2) \stackrel{G}{\longrightarrow} S U(3),
$$

and integrating along the fibres of the projection to $\mathfrak{M}$ :

$$
\mathscr{J}=p_{1 *} G^{*} \Theta .
$$

Consider now the diagram [with $G_{(n)}$ denoting the map $G$ on $\left.\mathfrak{M}^{(n)} \times S U(2)\right]$ :

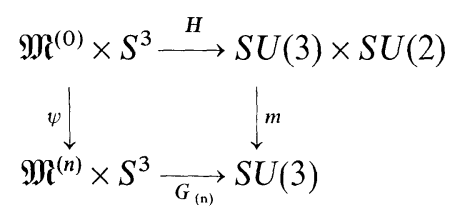

where $H(f, x)=(f(x), \mathbf{I}(x)), \Psi(f, x)=\left(f \mathbf{I}^{n}, x\right), m(a, b)=a b$.

From the Kunneth decomposition of $H^{*}(S U(3) \times S U(2))$ we see that $H^{*} m^{*} \Theta=G_{(0)}^{*} \Theta$. This gives

$$
\left[\Psi^{*} G_{(n)}^{*} \Theta\right]=\left[H^{*} m^{*} \Theta\right]=\left[G_{(0)}^{*} \Theta\right] .
$$

This proves the first statement.

Let us now look at $\mathscr{J}$ on $\mathfrak{M}^{(0)}$. We have $\mathscr{J}=p_{1^{*}} G_{(0)}^{*} \Theta$. If $\chi_{0} \mathscr{J}$ is a generator of $H^{2}\left(\mathfrak{M}^{(0)}\right)$ we have $\chi_{0} \mathscr{J}[h]=1$, where $h$ is a 2 -sphere generating $H_{2}\left(\mathfrak{M}^{(0)}\right)$ $=\pi_{2}\left(\mathfrak{M}^{(0)}\right)$. That is, $\chi_{0} G_{(0)}^{*} \Theta\left[p_{1}^{-1} h\right]=1$. But then it follows from the fact that $\pi_{2}\left(\mathfrak{M}^{(0)}\right) \sim \pi_{5}(S U(3))$ and the theorem on p. 237 of [12] that

$$
\chi_{0}=-\frac{i}{2 \pi} \frac{1}{240 \pi^{2}} \text {. }
$$

Remark 3.3. The $S U(2) \sigma$-model.

It is instructive to consider in parallel the $S U(2) \sigma$-Model. We summarise the relevant features: 
a) The configuration space, which we denote by $\mathfrak{N}$, splits up as in the $S U(3)$ case: $\mathfrak{N}=\bigcup \mathfrak{N}^{(n)}$. We have $\mathfrak{N}^{(m)} \hookrightarrow \mathfrak{M}^{(m)}$.

b) $\pi_{1}\left(\mathfrak{n}^{(m)}\right)=\mathbb{Z} / 2, \pi_{2}\left(\mathfrak{N}^{(m)}\right)=\mathbb{Z} / 2$. This implies that

$$
\operatorname{Ext}\left(H_{1}, \mathbb{Z}\right)=H^{2}\left(\mathfrak{N}^{(m)}\right)=\mathbb{Z} / 2 .
$$

[Sketch of proof: It is enough, by the universal coefficient theorem for cohomology and homology, to show $H^{2}$ is torsion. But if $\tilde{\mathfrak{N}}^{(n)}$ denotes the covering space

$$
H^{2}\left(\mathfrak{N}^{(m)}, \mathbb{R}\right) \hookrightarrow H^{2}\left(\tilde{\mathfrak{N}}^{(m)}, \mathbb{R}\right)=0 .
$$

The fact that the above map is an injection follows from [13].] We let $\mathscr{L}_{\mathfrak{N}}$ denote the nontrivial line bundle on $\mathfrak{N}^{(m)}$.

c) The curvature $\left.\mathscr{J}\right|_{\mathfrak{R}}=0$. This follows from Remark 2.1 .

\section{The Action of the Rotation Group, Spin}

We begin with a few general remarks. Let $L \rightarrow M$ be a hermitian line bundle on a manifold $M, F$ the curvature of a (unitary) connection $A$ on $L$, and $G$ a connected Lie group which acts on $M$ leaving $F$ invariant. Let $\varphi_{g}: M \rightarrow M$ denote the action of $g \in G$ on $M$ - we have $\varphi_{g} \circ \varphi_{g^{\prime}}=\varphi_{g g^{\prime}}$. Since $G$ is connected, each $\varphi_{g}$ has a lift, to $L$ :

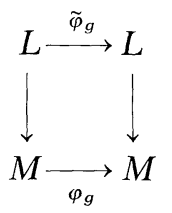

Note that $\tilde{\varphi}_{g}$ is not unique, for one can compose it with automorphisms of $L$ leaving $M$ fixed (gauge transformations). Under certain circumstances [for example if $H^{1}(M, \mathbb{R})=0$ or if $L$ is trivial and $F=0$ ] there exists a lift $\tilde{\varphi}_{g}$ which leaves $A$ invariant, and this is unique up to a constant gauge-transformation. Thus one has central extension:

$$
1 \rightarrow U(1) \rightarrow \hat{G} \rightarrow G \rightarrow 0 .
$$

Here $\hat{G}$ is a group of automorphisms of $L$ preserving $A, U(1)$ is a central subgroup of $G$ and $G=\hat{G} / U(1)$.

In general this will be a nontrivial extension - there may not be a way of choosing the lifts $\tilde{\varphi}_{g}$ s.t. $\tilde{\varphi}_{g} \tilde{\varphi}_{g^{\prime}}=\tilde{\varphi}_{g g^{\prime}}, \forall g, g^{\prime} \in G$. However, if $G=S U(n)$, and all actions are continuous the extension is trivial [14]. Note that this means if $G=S U(n) /($ centre), its action lifts to an action of $S U(n)$. We wish to prove:

Theorem 2. The action of the group of spatial rotations on $\mathfrak{M}^{(n)}$ (respectively $\mathfrak{N}^{(n)}$ ) for $n$ odd does not lift to $\mathscr{L}^{(j)}$ for $j$ odd (respectively to $\mathscr{L}_{\mathfrak{R}}$ ).

*Theorem 2. The action lifts otherwise.

Recall that $\mathscr{L}^{(j)}$ is the line-bundle with Chern class equal to $j$ times the generator of $H^{2}\left(\mathfrak{M}^{(n)}\right)$ and $\mathscr{L}_{\mathfrak{R}}$ is the nontrivial line bundle on $\mathfrak{N}^{(n)}$. 
Remark 4.1. Under the identification $S^{3} \sim S U(2)$, the group of spatial rotations can be identified with the inner automorphisms. The point $e$, left fixed, is the origin.

We shall first obtain a finite-dimensional manifold $M$, with a submanifold $N$ such that we have a commutative diagram of inclusions

$$
\begin{aligned}
& N \hookrightarrow \mathfrak{N}^{(1)} \\
& \checkmark \quad \curvearrowleft \\
& M \hookrightarrow \mathfrak{M}^{(1)}
\end{aligned}
$$

We will prove $H^{2}\left(\mathfrak{M}^{(1)}\right) \underset{\sim}{\sim} H^{2}(M) \rightarrow H^{2}(N) \longleftarrow H^{2}\left(\mathfrak{N}^{(1)}\right)$.

(The double arrow $\rightarrow$ will denote a surjection.)

Let us first define $M$. The group $S U(3)$ acts on $\mathfrak{M}^{(1)}$ by $(g, \psi) \rightarrow g \psi g^{+}$. The orbit of $\mathbf{I}$ under this action is defined to be $M$. Note that we have a diagram

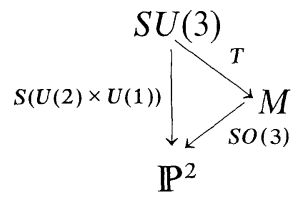

where $T$ is the $U(1)$ subgroup of $S U(3)$ consisting of matrices of the form

$$
\left(\begin{array}{ccc}
\alpha & 0 & 0 \\
0 & \alpha & 0 \\
0 & 0 & \frac{1}{\alpha^{2}}
\end{array}\right), \alpha \in U(1) .
$$

(This is the centraliser of $\mathbf{I}$.) We let $N=S U(2)$-orbit of $\mathbf{I}=$ fibre above the point $(0,0,1)$ of $\mathbb{P}^{2}$. Note that the inclusion in (4) are clear.

\section{Lemma 4.2.}

$$
\begin{array}{cl}
\pi_{1}(M)=0, & \pi_{2}(M)=\mathbb{Z}, \\
\pi_{1}(N)=\mathbb{Z} / 2, & \pi_{2}(N)=\mathbb{Z} / 2, \\
\mathbb{Z}=H^{2}(M) \rightarrow H^{2}(N)=\operatorname{Ext}\left(\pi_{1}(N), \mathbb{Z}\right)=\mathbb{Z} / 2 .
\end{array}
$$

Proof. Note $N \sim S O(3)$. The rest of the proof follows by looking at the homotopy exact sequence of the two fibrations. Only $\left({ }^{*}\right)$ requires comment. Note that we have

$$
0 \rightarrow \pi_{2}(M) \rightarrow \pi_{2}\left(\mathbb{P}^{2}\right) \rightarrow \pi_{1}(N) \rightarrow 0 .
$$

Applying $\operatorname{Hom}(\cdot, \mathbb{Z})$ to this we get

$$
0 \leftarrow H^{2}(N)=\operatorname{Ext}\left(\pi_{1}(N), \mathbb{Z}\right) \leftarrow H^{2}(M) \leftarrow H^{2}\left(\mathbb{P}^{2}\right) \leftarrow 0 .
$$

Proposition 4.3. We have

$$
\begin{aligned}
& \pi_{1}(N) \underset{\sim}{\sim}\left(\pi_{1}(\mathfrak{N})\right. \\
& \pi_{2}(M) \underset{\sim}{\sim}\left(\pi_{2}(\mathfrak{M}),\right. \\
& H^{2}\left(\mathfrak{M}^{(1)}\right) \underset{\sim}{\longrightarrow} H^{2}(M) \rightarrow H^{2}(N) \longleftarrow H^{2}\left(\mathfrak{N}^{(1)}\right) .
\end{aligned}
$$


Proof. It now suffices to prove (A) and (B). The idea is to take generators of the homotopy groups $\pi_{1}(N)$ and $\pi_{2}(M)$ and to show that they give generators of $\pi_{4}(S U(2))$ and $\pi_{5}(S U(3))$ respectively. Let us first prove (A). The path

$$
t \mapsto\left(\begin{array}{cc}
e^{i t \pi} & 0 \\
0 & e^{-i t \pi}
\end{array}\right) \equiv \eta(t), \quad t \in[0,1]
$$

in $S U(2)$ descends to a loop in $N$, the generator of $\pi_{1}(N)$. We have thus a diagram

$$
\begin{aligned}
& S U(2) \times S^{1} \\
& \uparrow \\
& S U(2) \times[0,1] \rightarrow S U(2) \\
& (g, t) \mapsto \eta^{+}(t) g \eta(t)
\end{aligned}
$$

The map $S U(2) \times S^{1} \rightarrow S U(2)$ is the $2 \pi$-rotation of $S U(2)=S^{3}$ and it is well-known that this gives a generator of $\pi_{4}\left(S^{3}\right)$ under the identification: $\pi_{4}\left(S^{3}\right)$ $\sim \pi_{1}\left(\operatorname{Maps}\left(S^{3}, S^{3}\right)\right)$, where on the right we take the identity map as base-point.

The proof of $(B)$ takes a little more work and we relegate it to an appendix.

Remark 4.4. We have the diagram

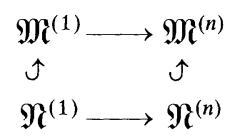

where the horizontal maps are by multiplying by powers of $\mathbf{I}$, and hence isomorphisms. We can thus conclude $H^{2}\left(\mathfrak{M}^{(n)}\right) \rightarrow H^{2}\left(\mathfrak{M}^{(n)}\right)$.

Remark 4.5. Define $N^{(n)}$ to be the $S U(2)$ orbit of $\mathbf{I}^{n}(n \neq 0)$. (Thus $N=N^{(1)}$.) Then the proof of (A) above can be imitated to show that $H^{2}\left(\mathfrak{N}^{(n)}\right) \rightarrow H^{2}\left(N^{(n)}\right)$ is an isomorphism for $n$ odd, and the zero map for $n$ even. We can now proceed with the

Proof of Theorem 2. Note that the action of the rotation group leaves $\mathfrak{N}^{(n)}$ invariant. By Remark 4.3 it suffices to prove that the action on $\mathfrak{N}^{(i)}$ for $i$ odd does not lift to $\mathscr{L}_{\mathfrak{N}}$. By Remark 4.4, it suffices to prove it for $N^{(i)}$ (noting that $N^{(i)}$ is also invariant under the rotation group). But under the identification $N^{(i)} \sim S O(3)$, it only remains to remark that the left action of $S O(3)$ on itself does not lift to the nontrivial line-bundle on it.

Proof of *Theorem 2. We know that the action lifts to a $S U(2)$ action. But by Remarks 4.3 and 4.4 it is clear that the element $\left(\begin{array}{rr}-1 & 0 \\ 0 & -1\end{array}\right)$ acts trivially on the fibres above $N^{(n)}$, and hence everywhere. For $n=0$ note there exists a point of $\mathfrak{M}^{(0)}$ left fixed by $S O(3)$. Then $S U(2)$ has to act trivially on the fibre above this point. (Concluding) Remark 4.5. In the published version of [1], which appeared after this work was completed, Witten considers the space $M$ and remarks that the Wess-Zumino term can be regarded as a "magnetic field" on it. The monopole harmonics are sections of the line-bundle $\mathscr{L}^{(3)}$ restricted to $M$. Since this is induced by a representation of $T$, the Frobenius reciprocity theorem can be applied to give information on the representations of $S U(3)$ that appear. 
Appendix: Proof of Proposition 4.2(B): We outline the proof in steps:

i) Define a map $K: S U(2) \rightarrow S U(2)$ as follows. If $a=\left(\begin{array}{rr}a & b \\ -\bar{b} & \bar{a}\end{array}\right) \in S U(2)$ (which is true iff $|a|^{2}+|b|^{2}=1$ )

$$
K u=\frac{1}{\left(|a|^{4}+|b|^{4}\right)^{1 / 2}}\left(\begin{array}{rr}
a^{2} & -\bar{b}^{2} \\
b^{2} & \bar{a}^{2}
\end{array}\right) .
$$

If $H$ is the torus $\left\{\left(\begin{array}{ll}\alpha & 0 \\ 0 & \bar{\alpha}\end{array}\right), \alpha \in U(1)\right\}$, we have

$$
K\left[\left(\begin{array}{cc}
\alpha & 0 \\
0 & \bar{\alpha}
\end{array}\right) u\right]=(K u)\left(\begin{array}{cc}
\alpha^{2} & 0 \\
0 & \bar{\alpha}^{2}
\end{array}\right)
$$

and $K$ induces a map $\hat{K}$ :

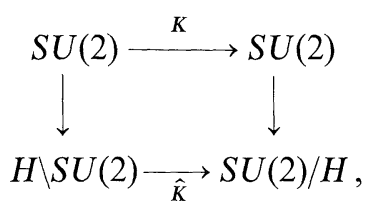

where $\hat{K}$ is of degree 2 .

ii) Define $\mathbf{J}: S U(2) \rightarrow S U(3)$ by $\mathbf{J} u=\left(\begin{array}{c|cc}1 & 0 & 0 \\ \hline 0 & K u \\ 0 & K u\end{array}\right)$. Then the map $S U(2) \stackrel{\mathbf{J} \cdot \mathbf{I}}{\longrightarrow} S U(3), u \mapsto \mathbf{J} u \cdot \mathbf{I} u$ satisfies

$$
\left(\begin{array}{cc}
\alpha & 0 \\
0 & \bar{\alpha}
\end{array}\right) u \mapsto(\mathbf{J} u \cdot \mathbf{I} u)\left(\begin{array}{ccc}
\alpha & 0 & 0 \\
0 & \alpha & 0 \\
0 & 0 & \bar{\alpha}^{2}
\end{array}\right)
$$

and thus induces a map $H \backslash S U(2) \stackrel{\Sigma}{\longrightarrow} M$. The induced map $H \backslash S U(2) \rightarrow \mathbb{P}^{2}$ is given by $u \mapsto \mathbf{J} u\left(\begin{array}{l}0 \\ 0 \\ 1\end{array}\right)$ and represents twice the generator of $\pi_{2}\left(\mathbb{P}^{2}\right)$. Thus $\Sigma$ represents a generator of $\pi_{2}(M)$.

iii) We shall prove (the square-brackets indicating homotopy classes of maps)

$$
\left[S^{2}, M\right] \rightarrow\left[S^{2}, \mathfrak{M}^{(1)}\right] \underset{T^{-1}}{\longrightarrow}\left[S^{2}, \mathfrak{M}^{(0)}\right] \rightarrow\left[S^{5}, S U(3)\right]
$$

gives an isomorphism $\pi_{2}(M) \sim \pi_{5}(S U(3))$. Let $v$ denote the vector

$$
\left(\begin{array}{l}
0 \\
0 \\
1
\end{array}\right) \in \mathbb{C}^{3}
$$


The map $S U(3) \rightarrow S^{5}(h \in S U(3) \mapsto h v)$ takes a generator of $\pi_{5}(S U(3))$ to twice a generator of $\pi_{5}\left(S^{5}\right)$. One can verify that it thus suffices to prove that in the diagram

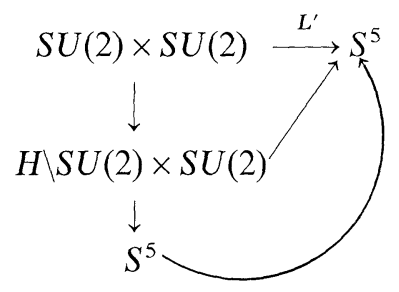

the map $L$ is of degree \pm 2 . Here $L^{\prime}$ is defined by

$$
L^{\prime}(u, g)=\mathbf{J} u \mathbf{I} u \mathbf{I} g(\mathbf{I} u)^{+}(\mathbf{J} u)^{+} v .
$$

iv) That $L$ is of degree \pm 2 will follow from the following fact: Let (B) denote the ("bad") set $(e) \times S U(2) \cup(-e) \times S U(2) \cup(H \backslash S U(2)) \times e$ in $H \backslash S U(2) \times S U(2)$, where $(e)$ denotes the $H$-coset $\left(\begin{array}{ll}1 & 0 \\ 0 & 1\end{array}\right)$ and $(-e)$ that of $\left(\begin{array}{rr}-1 & 0 \\ 0 & -1\end{array}\right)$. Then: a) outside (B) the differential of $L$ is nonzero b) outside the image of (B) every point has two pre-images, and c) the complement of $(\mathrm{B})$ is connected.

v) Let $w=(\mathbf{J} u)(v)$. Then

$$
(w, L(u, g))=(w, v),
$$

where (,) denotes the inner product in $\mathbb{C}^{3}$.

The claims a), b), c) in iv) above follow from (\#) and a rather tedious analysis of possibilities, which we omit.

It is possible to give a slightly shorter proof using $\left.\mathscr{J}\right|_{M}$ and the theorem of [12] that is used in the proof of Theorem 1.

Acknowledgements. It is a pleasure to thank A. Dhar, P. P. Divakaran, M. S. Narasimhan, M. S. Raghunathan, and S. Wadia for conversations regarding this work.

\section{References}

1. Witten, E.: Global aspects of current algebra. Nucl. Phys. B 223, 422 (1983)

2. Witten, E.: Current algebra, baryons, and quark confinement. Nucl. Phys. B 223, 433 (1983)

3. Finkelstein, D., Rubinstein, J.: Connection between spin, statistics, and kinks. J. Math. Phys.9, 1762 (1968)

4. Friedman, J.L., Sorkin, R.D.: Kinematics of Yang-Mills Solitons. Commun. Math. Phys. 89, $501(1983)$

5. Friedman, J.L., Sorkin, R.D.: Statistics of Yang-Mills solitons. Commun. Math. Phys. 89, 501 (1983)

6. Asorey, M., Mitter, P.: Ref. TH 3424-CERN

7. Balachandran, A.P., et al.: Soliton States in the quantum-chromodynamic effective Lagrangian. Phys. Rev. D 27, 1153 (1983) 
8. Dhar, A., Wadia, S.: The Nambu Jona-Lasinio model: an effective largangian for quantum chromodynamics at intermediate length scales. TIFR preprint, 1983

9. Sternberg, S.: Minimal coupling and the symplectic mechanics of a particle in the presence of a Yang-Mills field. Proc. Natl. Acad. Sci. USA 74, 5235 (1977)

10. Simms, D.J., Woodhouse, N.M.J.: Lectures on geometric quantisation. Berlin, Heidelberg, New York: Springer (Lecture Notes in Physics) 1976

11. Switzer, R.M.: Algebraic topology homotopy and homology. Berlin, Heidelberg, New York: Springer 1975

12. Bott, R., Seeley, R.: Some remarks on a paper of Callias. Commun. Math. Phys. 62, 235 (1978)

13. Seminaire, H., Cartan, E.N.S., 3e annee: 1950/51 ( $2^{\mathrm{e}}$ edition, multigraphiée).: Cohomologie des groupes, suites spectrale, faisceaux

14. Varadarajan, V.S.: Geometry of the quantum theory. New York: Van Nostrand Reinhold 1970

Communicated by R. Stora

Received October 23, 1983 
